\title{
Approche ergonomique de l'usage du régulateur de vitesse conventionnel : analyse critique de la littérature scientifique
}

An ergonomic approach to conventional cruise control use: a critical analysis of the scientific literature

\section{Samuel Aupetit et Jacques Riff}

\section{OpenEdition \\ Journals}

Édition électronique

URL : http://journals.openedition.org/activites/2413

DOI : 10.4000/activites.2413

ISSN : $1765-2723$

Éditeur

ARPACT - Association Recherches et Pratiques sur les ACTivités

\section{Référence électronique}

Samuel Aupetit et Jacques Riff, « Approche ergonomique de l'usage du régulateur de vitesse

conventionnel : analyse critique de la littérature scientifique », Activités [En ligne], 7-2 | octobre 2010

mis en ligne le 15 octobre 2010, consulté le 30 avril 2019. URL : http://journals.openedition.org/ activites/2413; DOI : 10.4000/activites.2413

\section{c.) (i) (9)}

Activités est mis à disposition selon les termes de la licence Creative Commons Attribution - Pas d'Utilisation Commerciale - Pas de Modification 4.0 International. 


\title{
Approche ergonomique de l'usage du régulateur de vitesse conventionnel : analyse critique de la littérature scientifique
}

\author{
Samuel Aupetit ${ }^{1}$ \\ Laboratoire Activité Motrice et Adaptation Psycho Physiologique (AMAPP), Université d'Orléans, 2 allée du Château, \\ BP 6237, 45062 Orléans Cedex 2, France \\ et Laboratoire Exploitation, Perception, Simulateurs et Simulations (LEPSIS), Institut National de Recherche sur les \\ Transports et leur Sécurité (INRETS) / Laboratoire Central des Ponts et Chaussées (LCPC), \\ 58 boulevard Lefebvre, 75731 Paris Cedex 15, France \\ aupetit@inrets.fr
}

\section{Jacques Riff}

Laboratoire Activité Motrice et Adaptation Psycho Physiologique (AMAPP), Université d'Orléans, 2, allée du Château, BP 6237; 4502 Orléans Cedex 2, France

jacques.riff@univ-orleans.fr

\begin{abstract}
An ergonomic approach to conventional cruise control use: a critical analysis of the scientific literature. This article offers a critical analysis of existing scientific literature on the use of conventional cruise control (CCC). This driver assistance system, which maintains a speed pre-set by the driver, is gradually being incorporated into all passenger cars in France. This review makes it possible to (1) determine the benefits and drawbacks of CCC for drivers, and the problems related to its use, (2) pinpoint the lack of systematic ergonomic studies concerning CCC use, despite the efforts of cruise control designers and car manufacturers to introduce the device in all market segments, and (3) suggest ways this research could be improved as regards the individual-group aspect of cruise control use, the conditions in which data are collected in both laboratory and real-life situations, and the static-dynamic aspect of learning to use CCC. This review has benefited from the latest advances in research into adaptive cruise control (ACC), a recent development of $\mathrm{CCC}$, which in the short time since it was launched has already given rise to a large amount of literature.
\end{abstract}

KEYwordS

Driving assistance system, ergonomics, conventional cruise control; situated action.

\section{Introduction}

Une des évolutions actuelle en matière automobile est le développement des technologies d'assistance au conducteur de plus en plus nombreuses et sophistiquées. Ainsi sont disponibles des systèmes d'affichage tête haute, des lecteurs avertisseurs de limitation de vitesse, des systèmes d'alerte de franchissement de ligne, des détecteurs de somnolence au volant, des dispositifs d'aide au stationnement, de surveillance d'angle mort, de vision infrarouge de nuit, des systèmes adaptant la vitesse dans les virages, d'aide au freinage, au repérage... Chacune de ces technologies est le plus souvent implantée selon le processus suivant: dans un premier temps au sein des véhicules de haut de gamme

1. Doctorant financé par la Fondation MAIF et la Région Centre 
puis progressivement sur tout ou partie des segments du marché.

Cette revue de questions porte sur le régulateur de vitesse « conventionnel » ou Conventional Cruise Control (CCC), un des systèmes d'assistance à la conduite automobile parmi les plus répandu en Europe. Ce dispositif, qui assiste le conducteur dans la gestion de la vitesse du véhicule - ou plus techniquement le « contrôle longitudinal du véhicule »- est apparu dès 1958 aux États-Unis. Il a connu une évolution majeure avec la mise au point du régulateur de distance «Adaptive Cruise Control » (ACC) qui assure partiellement l'automatisation de la vitesse du véhicule mais aussi de la distance intervéhiculaire. Cette évolution est disponible le plus souvent en option (pour un tarif de 1.000 à 2.600 euros en 2010) sur des modèles très haut de gamme. Ces dispositifs, comme tous les systèmes d'assistance, sont conçus pour avoir des effets positifs sur l'activité des conducteurs dans le sens d'une plus grande sécurité, d'un confort amélioré, d'une facilitation de la conduite (Malaterre, \& Saad, 1986). On comprend donc que les constructeurs automobiles et leurs sous-traitants réalisent, en amont de la mise sur le marché de ces systèmes, des études ergonomiques systématiques qui permettent d'en comprendre l'impact sur l'activité de conduite et de s'assurer que les objectifs « d'aide » sont bien atteints. Les spécialistes de l'ergonomie savent bien qu'il est assez courant qu'une innovation apportant un progrès incontestable sur une dimension de la situation, puisse, de façon concomitante, poser de nouveaux problèmes à l'usager qui n'existaient pas dans la situation initiale. Un dispositif «d'aide » peut, de ce fait générer de la "gène », ce qui est contraire aux objectifs des concepteurs (Theureau, \& Jeffroy, 1994). Il est nécessaire également, de poursuivre cet effort de vigilance ergonomique lorsque le système est diffusé à plus grande échelle, pour vérifier ses impacts sur les utilisateurs, sous la forme de « retours d'expérience ». Pour ce qui concerne les régulateurs de vitesse, on assiste au paradoxe suivant: l'usage du régulateur de vitesse de nouvelle génération, l'ACC, qui équipe une fraction infime du parc automobile a donné lieu à un effort significatif de recherche du côté des équipementiers, des constructeurs automobiles et des organismes de recherche. On dispose d'un corps de connaissances important aux plans qualitatif et quantitatif sur l'utilisation de ce système récent et les difficultés qu'il pose aux conducteurs. À l'inverse, l'usage du régulateur de vitesse « conventionnel » qui équipe une part déjà très importante et en constante augmentation du parc de véhicules, n’a pas fait l'objet d'un effort de même ampleur: les études empiriques restent rares.

Cette revue de question a pour objectifs (1) de proposer une analyse critique de la littérature scientifique existante sur l'usage du régulateur de vitesse « conventionnel », (2) de tirer profit des avancées des recherches portant sur l'ACC pour mener un effort de recherche plus approfondi sur l'usage du modèle conventionnel.

Cette analyse critique de la littérature porte sur: (1) les articles disponibles sur la base TRIS (Transportation Research Information Services) publiée par le TRB (Transportation Research Board) qui fait référence dans le domaine des transports, (2) les programmes de recherches portant sur les systèmes d'aide à la régulation de la vitesse, dont les plus importants sont AIDE, FESTA et EuroFOT, (3) les articles obtenus de façon complémentaire par interrogation des moteurs de recherche généralistes et l'examen des listes de références des articles recueillis en 1 et 2.

\section{1.- Les recherches portant sur l'usage du régulateur de vitesse conventionnel}

\section{1.- Le régulateur de vitesse conventionnel et son évolution : le régulateur de distance}

Le régulateur de vitesse « conventionnel ${ }^{2}$ » est un système d'assistance à la conduite automobile dont la fonction est de conserver une allure prédéfinie par le conducteur, sans que ce dernier n'ait

2. Pour une description du régulateur de vitesse conventionnel du point de vue mécanique, voir Givens (1975) et Kawano (1992).. 
à maintenir un appui sur la pédale d'accélération (Patterson, 1998). Deux formes d'instrumentation existent: soit la commande prend la forme d'une manette placée derrière le volant, sur le même plan que l'indicateur de direction (Figure 1), soit il s'agit de boutons situés directement sur le volant (Figure 2).

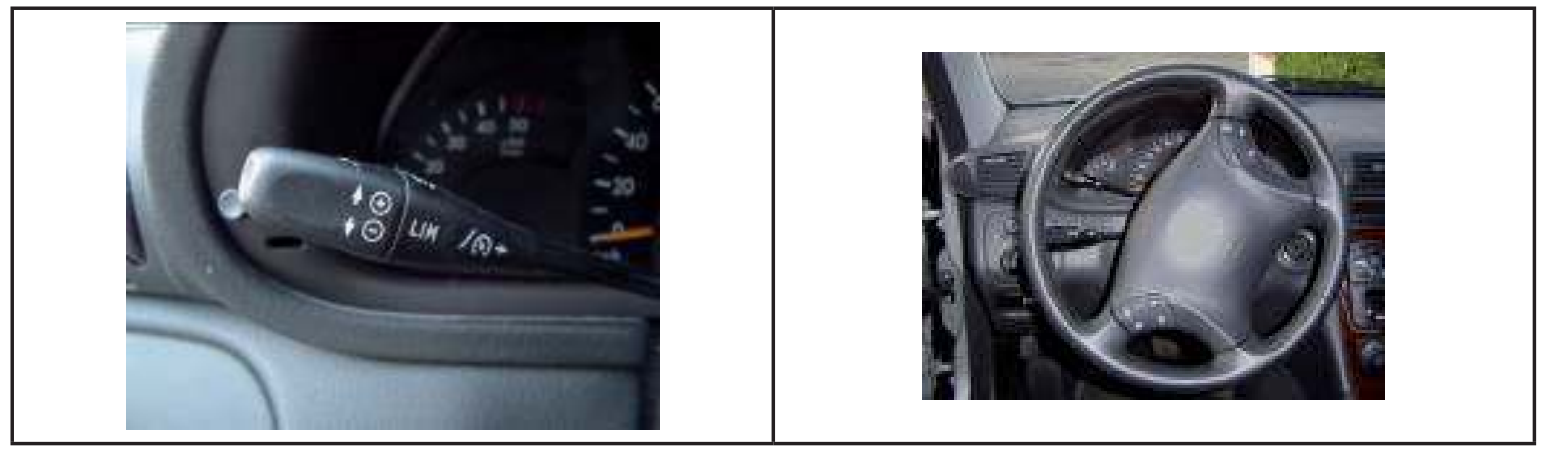

Figure 1. - Instrumentation « manette (Photos, constructeur allemand)

Figure 1. - Lever interface (Pictures, German car manufacturer)

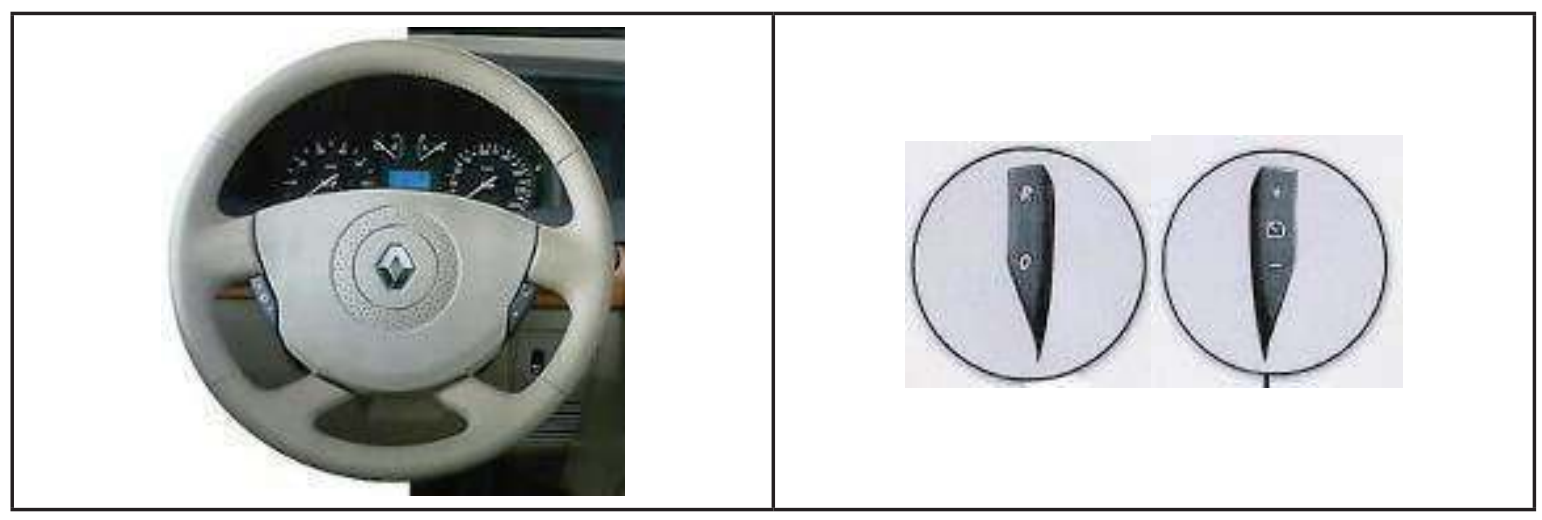

Figure 2. - Instrumentation « au volant» (Document Renault, 2004, modèle Renault Laguna)

Figure 2. - Steering wheel interface (Renault Document, 2004, Renault Laguna)

Une séquence d'utilisation type d'un régulateur de vitesse conventionnel comprend trois grandes phases: l'activation du dispositif, la fixation de la vitesse de croisière désirée et la déconnexion du système.

L'activation du dispositif se fait en actionnant l'interrupteur situé sur le tableau de bord (pour les instrumentations « manette » et « volant»).

La fixation de la vitesse de croisière désirée est effectuée en positionnant le véhicule à l'allure souhaitée (avec la pédale d'accélération), puis en enregistrant la vitesse pratiquée à l'aide de la touche «+- » pour l'instrumentation « volant» ou en actionnant vers le haut ou le bas la commande du système, pour l'instrumentation « manette ». S'il le souhaite le conducteur peut faire varier la vitesse enregistrée en utilisant le même type de commande (incrémentation des pas de 1 ou $2 \mathrm{~km} / \mathrm{h}$, selon les modèles). Il est à noter qu'une allure précédemment enregistrée en mémoire peut être « rappelée » en appuyant sur le bouton «Resume », noté « $\mathrm{R} »$ dans la figure 2 . Si la vitesse précédemment enregistrée est plus élevée que la vitesse courante, le véhicule accélère automatiquement (Patterson, 1998; Shaout, \& Jarrah, 1997).

La déconnexion du système peut se faire par différents moyens: appui sur la pédale de frein ou la pédale d'embrayage, positionnement du levier de vitesse au «point mort », enclenchement de l'in- 
terrupteur sur la position «Off », appui sur la touche « $\mathrm{O} »($ Figure 2). Cette dernière touche permet de désactiver instantanément le dispositif mais la vitesse de croisière reste mémorisée dans l'ordinateur de bord. Lorsque le dispositif est activé, le conducteur peut dépasser la vitesse enregistrée en appuyant sur la pédale d'accélération. En relâchant la pédale, le régulateur de vitesse « reprend le contrôle » et la vitesse du véhicule se rétablit progressivement au niveau de l'allure de croisière prédéfinie.

Le régulateur de vitesse « intelligent ${ }^{3}{ }$ nommé ACC (Adaptive Cruise Control) ou ICC (Intelligent Cruise Control) est l'évolution du régulateur de vitesse conventionnel. Couplé à un radar anti-collision, l'ACC assure partiellement l'automatisation du contrôle de la distance intervéhiculaire et de la vitesse de la voiture (Bachmann, Naab, Reichart, \& Scrault, 2000; Prestl, Sauer, Steinle, \& Tschernoster, 2000; Scapaticci, Fermi, \& Saroldi, 2000). L'ACC détecte la présence du véhicule précédent sur la voie, calcule l'allure de celui-ci et la distance qui sépare les deux véhicules (si celle-ci est inférieure à 150 mètres) (Saad, Hjälmdahl, Canas, Alonso, Garayo, Macchi, et al., 2004). La vitesse de la voiture est ensuite ajustée à celle du véhicule qui précède afin de maintenir une distance appropriée. Si ce véhicule roule plus lentement que la vitesse de croisière, l'ACC déclenche une décélération voire le freinage du véhicule. Si distance et vitesse relatives du véhicule précédent augmentent, le système provoque une accélération pour revenir au plus près de la vitesse de consigne. Lors d'un trafic faible ou nul, l'ACC fonctionne comme un régulateur de vitesse conventionnel. Dans certains cas d'approche d'un véhicule plus lent où le système détecte que sa capacité de décélération et de freinage ne seront pas suffisantes pour gérer la situation en toute sécurité, il déclenche une alerte indiquant au conducteur de reprendre la main (Villame, 2004). Le conducteur peut alors changer de voie ou accélérer. Le système peut être déconnecté en appuyant sur le frein, sur l'interrupteur ou lorsque l'allure du véhicule équipé est trop faible (généralement aux environs de $50 \mathrm{Km} / \mathrm{h}$ ). L'ACC peut également être suspendu momentanément et réactivé par la suite avec le bouton « Resume » (Szuszman, 2005).

\section{2.- Les recherches concernant exclusivement l'usage du régulateur de vitesse conventionnel}

Une revue récente du Centre de Recherche sur les Accidents de l'Université de Monash (Australie) recense les quelques d'études portant exclusivement sur le régulateur de vitesse conventionnel (Young, \& Regan, 2007). Elles s'intéressent principalement aux effets du régulateur de vitesse conventionnel sur la conduite et à l'appropriation de ce système.

Les effets du CCC sur le comportement de conduite. Une synthèse publiée par l'Institut Hollandais de Sécurité Routière (SWOW) montre que très peu de travaux portent sur les effets du régulateur de vitesse conventionnel sur la sécurité routière (Van Kampen, 1996). En mesurant la performance de conduite avec CCC et sans système d'assistance, ces études mettent en évidence les avantages de ce mode de régulation à plusieurs niveaux :

- Un gain en sécurité : la vitesse maintenue par le dispositif est une allure « sécuritaire » en adéquation avec les limites autorisées (Warren, 1982; Young, \& Regan, 2007), les temps intervéhiculaires (time headway) sont plus importants avec CCC que sans ${ }^{4}$ (Smith, Ashby, \& Fairclough, 1994), les distances intervéhiculaires sont perçues comme plus grandes (Saad, Dionisio, Arslanian, Collaciuri, Legay, Vézier, \& Timianguel, 2006), la vitesse estimée des conducteurs est en baisse (pour $70 \%$ des conducteurs étudiés dans Lheureux, Saad, Pianelli, Abric, \& Roland, 2006).

- Un gain en confort avec une diminution de la fatigue des membres inférieurs du conducteur, spécialement sur les longues distances, lui permettant d'adopter différentes positions de conduite (Callahan, 1992; Lheureux, et al., 2006; Young, \& Regan, 2007). L'intérêt du dispositif en ma-

3. Pour une synthèse des travaux portant sur ce dispositif, voir Saad et al. (2004).

4. Il faut noter que Smith et al. (1994) attribuent cette augmentation de temps intervéhiculaire à la diminution du sentiment de sécurité des participants au cours de la conduite avec régulateur de vitesse. 
tière de confort est même la principale raison de son utilisation pour $31 \%$ des conducteurs interrogés par Lheureux et al. (2006).

- Une diminution de la consommation de carburant (Lheureux, et al., 2006; Travis, 1982) liée à la diminution des à-coups.

— Une conduite plus « douce » (meilleure anticipation, diminution des dépassements risqués, moins de freinages accentués et de changements de vitesse...) (Saad, et al., 2006).

- Une diminution de la préoccupation relative aux contrôles de police (Lheureux, et al., 2006; Young, \& Regan, 2007). Pour Young et Regan (2007), c'est même la principale utilité du CCC. Les participants activent le dispositif quand le risque d'être contrôlé par la police est élevé. L'allure adoptée est en moyenne de $10 \mathrm{~km} / \mathrm{h}$ au-dessus de la vitesse autorisée. Ce choix semble être directement lié au seuil de tolérance des radars de police (Regan, \& Young, 2004).

- Le maintien de l'attention portée à la conduite (Christ, Smuc, Gatscha, Schmotzer, \& Otzelberger, 2000 ; Lheureux, et al., 2006). Christ et al.. (2000), qui ont étudié l'impact du CCC sur l'attention allouée à la tâche de conduite, montrent que les conducteurs $(\mathrm{N}=50)$ ne présentent ni altération de l'attention ni davantage de comportements de freinage non adaptés.

Certains effets négatifs de l'utilisation du CCC sur le comportement de conduite peuvent toutefois être distingués. Ils concernent notamment:

- La gestion des interactions avec les autres usagers de la route: gêner les autres conducteurs qui roulent à des allures irrégulières, difficulté qui s'accentue quand le trafic se densifie (Lheureux, et al., 2006) ;

- L'adoption de stratégies adaptatives de conduite qui peuvent présenter un risque relatif. Saad et al. (2006) rapportent que 7 conducteurs sur 12 ont prétendu conduire uniquement avec « les mains ». Au lieu d'appuyer sur la pédale d'accélérateur pour ajuster la vitesse du véhicule, ils utilisent les boutons «+- » du régulateur de vitesse ;

- L'effort cognitif et physique, mis en évidence par Young et Regan (2007), qui serait lié à l'ajustement permanent de la vitesse croisière en fonction des conditions de circulation rencontrées.

L'appropriation $d u C C C$. Des précisions sur l'appropriation du régulateur de vitesse conventionnel ont été rendues disponibles grâce à deux études récentes (Lheureux, et al., 2006; Saad, et al., 2006) menées dans le cadre du projet européen AIDE $^{5}$ (2004-2008) et Young et Regan (2007). L'étude de Saad et al. (2006) porte sur le processus d'apprentissage à court terme de l'utilisation du CCC de 12 conducteurs, qui n'avaient jamais conduit de véhicule muni de ce système. Trois sessions de conduite avec CCC ont été mises en place. Deux sessions d'une journée placées avant et après une semaine de conduite «autonome » avec un véhicule équipé prêté par les expérimentateurs. Au cours de cette semaine, le conducteur réalise ses trajets habituels. Le dispositif méthodologique combine observations du comportement du conducteur et entretiens effectués avant et après les différentes sessions (48 entretiens au total). La recherche menée par Lheureux et al. (2006) porte, elle, sur les effets à long terme de l'usage du CCC sur le comportement de conduite. Des questionnaires ont été renseignés par 236 clients d'un constructeur français, possédant un véhicule muni de CCC. Dans Young et Regan (2007), les données sont issues de questionnaires et d'entretiens réalisés avec 31 conducteurs australiens, avant et après le visionnage d'une vidéo explicative du « bon usage » du CCC.

L'appropriation du régulateur de vitesse conventionnel semble pouvoir être décomposée en trois phases (Saad, et al., 2006). Une première phase d'exploration qui consiste à apprendre à dialoguer avec le système et comprendre ses fonctions. Une deuxième où le conducteur teste les procédures et les limites du dispositif et commence à définir des contextes d'utilisation privilégiés. Enfin une dernière où il s'agit pour le conducteur de trouver le meilleur compromis entre procédures et contextes de conduite. Même si plusieurs participants ont connu des difficultés de familiarisation avec le système (Saad, et al., 2006; Young, \& Regan, 2007), tous ont réussi à maîtriser les principales fonc-

5. Le projet européen AIDE (2004-2008) a pour objectif de développer des méthodologies et des interfaces homme/machine destinés à l'intégration de dispositifs technologiques dans l'automobile. 
tionnalités du dispositif rapidement. Le temps de familiarisation avec CCC est ainsi estimé entre une heure et une heure trente (Saad, et al., 2006). La grande majorité (94\%) des conducteurs étudiés par Lheureux et al. (2006) déclare également que le dispositif a été appris facilement ou assez facilement et $65 \%$ d'entre eux qu'il a été maîtrisé après quelques utilisations. Selon ces auteurs, le principal vecteur de familiarisation est le commercial des concessions automobiles (pour $66 \%$ des utilisateurs interrogés). Son rôle est d'autant plus important que plus de la moitié des conducteurs étudiés n'ont pas lu la notice d'utilisation. Près de la moitié des usagers prétendent avoir appris seuls et quelques conducteurs déclarent avoir appris avec l'aide d'un ami.

L'analyse des résultats fait apparaitre plusieurs types de difficultés au cours de l'appropriation: (a) les conducteurs prétendent que les notices d'utilisation ne donnent pas les informations suffisantes pour comprendre le fonctionnement du CCC (Saad, et al., 2006), (b) les utilisateurs ont des problèmes pour apprendre à faire cohabiter une conduite à allure régulée et leurs habitudes de conduite (Young, \& Regan, 2007), (c) la maîtrise des différentes manières de suspendre et désactiver le CCC nécessitent un temps d'apprentissage conséquent (Saad, et al., 2006), (d) la majorité des conducteurs ont des difficultés pour assimiler les procédures de réactivation du système (les boutons « $\mathrm{R}$ » ou «+-») (Saad, et al., 2006), (e) l'essentiel des participants, dans Saad et al. (2006), sont préoccupés par l'identification des situations de conduite où ils doivent désactiver le système et reprendre le contrôle manuel du véhicule. L'ensemble de ces difficultés semblent présentes pour les conducteurs étudiés au cours des premières expériences de conduite régulée et occasionner un sentiment de peur chez les usagers: Lheureux et al. (2006) indiquent que près d'un conducteur sur trois s'est fait peur plusieurs fois lors des premières utilisations du système. Le dispositif méthodologique utilisé (question fermée) ne donne malheureusement pas la possibilité de définir plus précisément le contexte de l'émergence de cette émotion et de caractériser les situations génératrices de peur. L'ensemble de ces difficultés d'appropriation conduirait un certain nombre de conducteurs possédant un véhicule équipé du CCC à ne pas utiliser ce dispositif (Lheureux, et al., 2006). Au bilan, les travaux évoqués sont parvenus à identifier les effets positifs et négatifs de l'utilisation du régulateur de vitesse conventionnel sur le comportement de conduite, spécialement en termes de confort et de sécurité. Les travaux les plus récents (Lheureux, et al., 2006; Saad, et al., 2006; Young, \& Regan, 2007) apportent, eux, des éléments fondamentaux de compréhension de l'appropriation de ce système. On peut toutefois remarquer le décalage entre le nombre de travaux scientifiques disponibles au plan international qui traitent en profondeur et de manière spécifique des questions d'appropriation et d'usage du régulateur de vitesse conventionnel et l'omniprésence de ce système dans les véhicules actuels. Malgré les efforts du projet AIDE et Young et Regan (2007), on ne dispose encore pas d'un corpus de données suffisamment important pour décrire les mécanismes effectifs d'utilisation et d'appropriation du dispositif en contexte réel.

\section{3.- Les recherches sur l'usage du régulateur de vitesse conventionnel en comparaison avec le régulateur de distance}

La nouvelle génération de régulateur de vitesse, dite « intelligente » (Adaptive Cruise Control noté ACC), réservée à quelques voitures haut de gamme, fait l'objet de nombreux travaux (voir Ioannou, \& Stefanovic, 2005; Ma, \& Kaber, 2005; Rajaonah, Anceaux, \& Vienne, 2006 notamment $\left.^{\circ}\right)$. La performance de conduite avec régulateur de vitesse conventionnel apparaît comme une valeur de référence pour tester l'efficacité de la nouvelle génération du dispositif (par exemple : Fancher, Baraket, Johnson, \& Sayer, 1995 ; Jenness, Lerner, Mazor, Osberg, \& Tefft, 2008 ; Koziol, Inman, Carter, Robinson, \& Barker, 1999; Rakha, Hankey, Patterson, \& Van Aerde, 2001; Sayer, Mefford, Shirkley, \& Lantz, 2005; Schober, Eskandarian, Kühne, \& Wehlan, 2004; Turrentine, Sperling, \& Hungerford, 1991; Watanabe, Kishimoto, Hayafune, \& Yamada, 1995 ; Youngbin, 1997). En mesurant les progrès réalisés par le dispositif le plus récent et comparant la performance de conduite $\mathrm{CCC} /$ ACC, ces travaux offrent de précieuses informations sur le comportement de conduite avec le modèle

6. Pour une revue de littérature complète sur l'ACC, voir Saad et al. (2004). 
conventionnel. Ces études s'intéressent plus particulièrement à l'acceptabilité des deux modèles, aux effets de l'utilisation des systèmes sur le comportement de conduite en conditions de suivi (vitesse, distance et temps intervéhiculaires...) et l'impact de l'utilisation respective de ces deux dispositifs sur la charge mentale subie par le conducteur ( driver workload $\left.{ }^{7} »\right)$

L'acceptabilité des deux modèles. Dans Koziol et al. (1999), les participants $(N=108)$ ont conduit des véhicules équipés de CCC et d'ACC sur différents types de routes. Les données suggèrent que les conducteurs choisissent d'utiliser l'ACC plus souvent (50\%) que le modèle conventionnel pour des raisons de sécurité, de confort et d'utilisabilité. Au cours d'un même parcours, l'ACC est utilisé $55 \%$ du temps contre $40 \%$ avec le CCC (Rakha, et al., 2001). Les conducteurs préfèrent utiliser l'ACC car ils jugent l'activation et la désactivation du dispositif traditionnel trop coûteuses, inconfortables (Youngbin, 1997) et ils ont un sentiment accru de sécurité avec le dispositif récent (distances de sécurité perçues comme plus importantes et charge mentale du conducteur réduite avec ACC) (Fancher, et al., 1995).

Les effets sur la conduite en conditions de suivi. Ces études comparent le comportement de conduite avec CCC, ACC et sans système, en conditions de suivi d'un autre véhicule. La majorité de ces travaux montre que la distance et le temps intervéhiculaires sont moins importants avec CCC qu'avec ACC (Fancher, \& Ervin, 1994; Fancher, et al.., 1995; Koziol, et al., 1999, pour les principaux). Fancher et al. (1995) notent que les conducteurs utilisant le modèle conventionnel ont tendance à se trouver fréquemment très près (en distance et en temps) du véhicule suivi. Selon Fancher et Ervin (1994), l'utilisation du CCC entraîne inévitablement de faibles distances de suivi, car le conducteur doit estimer, dans chaque situation, la possibilité de maintenir sa vitesse de croisière, stratégie moins coûteuse. Koziol et al. (1999) montrent également que la vitesse moyenne des véhicules munis du modèle conventionnel est plus élevée qu'avec ACC en cas de freinage brusque du véhicule suivi. À l'opposé, un rapport récent de Jenness et al. (2008) pour la NHTSA indique que $38 \%$ des conducteurs prétendent se sentir plus en sécurité avec ACC qu'avec CCC (7\% dans le cas contraire).

La relation entre la charge mentale du conducteur et l'utilisation des dispositifs. L'objectif ici est de montrer en quoi la charge mentale est modifiée avec l'usage du CCC ou de l'ACC. Deux paramètres sont mesurés : le nombre d'interventions sur la pédale de frein associé à l'utilisation du système et le nombre d'appui sur les commandes du dispositif. Pour ce qui est du premier paramètre, les résultats sont controversés. Fancher et al. (1995) ont analysé le comportement de freinage à $88 \mathrm{Km} / \mathrm{h}$ de 36 conducteurs et concluent à une différence significative dans le nombre moyen d'interventions sur la pédale de frein suivant les dispositifs: 11,3 avec CCC, 7,4 avec ACC et 5,8 pour la conduite sans système. En revanche, aucune différence liée à l'utilisation du CCC ou de l'ACC n'a été identifiée pour les 108 participants de l'étude menée par Rakha et al. (2001).

Concernant le second paramètre, les résultats sont plus convergents. La plupart des travaux parviennent à montrer une réduction de la charge mentale du conducteur avec ACC par rapport au CCC (Rakha, et al., 2001; Stanton, Young, \& McCaulder, 1997; Watanabe, et al., 1995). Par exemple, Watanabe et al. (1995) ont mesuré les effets de l'introduction de CCC et d'ACC sur la charge mentale à partir du nombre d'interventions sur la commande «+- » servant à faire varier la vitesse de croisière et sur le bouton « Resume ${ }^{8} »$. Les résultats indiquent une réduction sensible de la charge mentale du conducteur avec ACC par rapport au dispositif conventionnel. Pour Rakha et al. (2001), la diminution de la charge mentale s'accompagne d'une réduction des demandes manuelles et visuelles de la tâche de conduite (pour $100 \mathrm{~km}, 15,3$ appuis sur la commande «+- » avec ACC et 16,3 fois avec CCC). Toutefois, pour Nilsson (1995) et Young et Stanton (1997), la charge totale de conduite est identique entre une conduite avec et sans système d'assistance. La conduite avec ACC occasionne une diminution de la tâche physique du conducteur en lui évitant d'appuyer sur la pédale d'accélération, mais complexifie la tâche cognitive par une activité de vérification du comportement

7. La « charge mentale » du conducteur désigne la partie cognitive de la charge de travail associée à la conduite (De Waard, 1996).

8. Le bouton « Resume » permet de « rappeler » une vitesse précédemment enregistrée en mémoire. 
du véhicule. Au lieu de freiner en cas de véhicule détecté avec un CCC, le conducteur avec ACC doit vérifier que le système a bien pris en compte le véhicule qui précède, que la voiture freine effectivement et que le dispositif maintient une distance appropriée. Pour Stanton et Young (2005), l'ACC permet de réduire la charge physique du conducteur, comparé à une conduite avec CCC, mais augmente, en contrepartie, sa charge mentale. Sayer et al. (2005) mettent d'ailleurs en lumière un nombre moins élevé de comportements de distraction avec CCC qu'avec ACC au cours d'une étude menée en conditions de trafic réel.

Au final, l'ensemble des travaux présentés montre que l'ACC a progressé en matière de confort d'utilisation par rapport au modèle conventionnel. En matière de sécurité les avancées sont plus contrastées: distance de sécurité allongée, vitesse moyenne moins élevée, mais temps de réaction plus important qu'avec CCC. Les gains apportés par l'utilisation de l'ACC par rapport au CCC sur la charge de conduite n'ont pas été uniformément prouvés. L'hypothèse d'une activité cognitive de vigilance à l'égard du bon fonctionnement de l'ACC est avancée. Ces travaux ont le mérite d'apporter des éléments de précisions importants sur la conduite avec régulateur de vitesse conventionnel et viennent compléter les résultats présentés précédemment.

\section{2.- Perspectives d'enrichissement des études portant sur l'usage du régulateur conventionnel}

Les travaux portant sur le régulateur de vitesse conventionnel peuvent, selon nous, être enrichis en tirant profit des avancées théoriques et méthodologiques de la recherche sur l'ACC par l'intégration de trois orientations qui concernent (1) la dimension individuelle-collective de l'usage du régulateur, (2) les conditions de recueil des données laboratoire-situations réelles et (3) le caractère statique-dynamique de l'appropriation du CCC.

\section{1.- La dimension individuelle-collective de l'usage du régulateur de vitesse conventionnel}

La majorité des travaux portant sur l'utilisation du CCC adopte un niveau d'analyse centré sur les interactions conducteur/dispositif, privilégiant l'idée d'un utilisateur « solitaire » plus ou moins déconnecté de son environnement social. Or il existe une littérature significative qui souligne l'importance de la dimension collective dans les activités de conduite et distingue une dimension collective dite « intravéhiculaire », caractérisant les phénomènes sociaux qui concernent des personnes présentes au sein de l'habitacle, d'une dimension "extravéhiculaire », désignant les phénomènes sociaux qui concernent la cohabitation entre le conducteur et d'autres usagers de la route. Les premières, menées dans des perspectives théoriques multiples [d'accidentologie, comportementaliste ou sociologique, voir Regan et Mitsopoulos (2003) pour une analyse plus poussée des recherches sur cette thématique] décrivent les comportements d'ajustement des conducteurs aux attentes des passagers (Baxter, et al., 1990), tendent à considérer à considérer les passagers comme une source de distraction pour le conducteur (Goodman, Tijerina, Bents, \& Wierwille, 1999; Lee, Caven, Haake, \& Brown, 2001; Wang, Knipling, \& Goodman, 1996; Zaidel, Paarlberg, \& Shinar, 1978) ou, à l'inverse, soulignent l'aide active des passagers pour orienter le conducteur, répondre au téléphone à sa place, ajuster les dispositifs de confort, le maintenir en éveil, l'alerter d'un danger, commenter la vitesse adoptée... (Regan, \& Mitsopoulos, 2003).

Les travaux disponibles relatifs à la dimension collective « extravéhiculaire » caractérisent la conduite automobile comme une activité mobilisant plusieurs usagers de la route en interactions constantes (Peretti-Watel, 2001). Pour Keegan (1996), la conduite nécessite la mobilisation de « compétences sociales » qui permettent d'évoluer dans un environnement complexe en s'articulant avec les autres usagers, les conducteurs sont sensibles au comportement des autres usagers dans la détermination de leurs modalités de conduite: le choix de la vitesse du véhicule dépend de l'allure des véhicules 
présents dans l'espace proche et de la densité de circulation (Connolly, \& Aberg, 1993; Holland, \& Conner, 1996). L'essentiel de ces travaux indique que la conduite automobile est une «activité sociale » (Barjonet, 1989; Peretti-Watel, 2001) largement contrainte par le comportement d'autrui.

Nous estimons que la tendance à considérer l'utilisation du régulateur comme le seul fait du conducteur, tendance que l'on observe dans la majorité des études portant sur le régulateur de vitesse conventionnel, aboutit à sous-estimer l'influence des passagers et celle des autres usagers de la route dans les situations d'usage du CCC. De plus, la considération d'un utilisateur « solo » apparaît en décalage par rapport à l'importance de la composante sociale mise en évidence par un certain nombre de travaux portant sur l'usage du régulateur de distance. Pourquoi les travaux sur l'ACC, qui soulignent le poids de cette dimension collective, ne pourraient-ils pas être étendus au CCC ? Saad et Villame (1996) et Fancher, Ervin, Sayer, Hagan, Bogard, Bareket, et al. (1998) mettent en lumière, par exemple, que l'évolution du trafic conditionne pour partie l'usage de l'ACC. À un autre niveau, plusieurs travaux (par exemple, Bjørkly, Jenssen, Moen, \& Vaa, 2003; Hoedemaeker, \& Kopf, 2001; Ojeda, \& Nathan, 2006 ; Rudin-Brown, \& Parker, 2004 ; Törnros, Nilsson, Östlund, \& Kircher, 2002) ont montré la manière dont l'usage de l'ACC contraignait les modes d'interactions du conducteur avec les autres usagers. D'autres études (notamment Saad, \& Malaterre, 1982; Saad, et al., 2006) indiquent que les usagers de dispositifs régulant la vitesse de conduite sont préoccupés par l'interprétation que peuvent faire les autres conducteurs de leurs propres comportements de conduite. Les systèmes n'auraient pas seulement des effets sur le comportement du conducteur " assisté » mais également sur les perceptions et la conduite des usagers au « contact », qu'ils soient équipés ou non du dispositif. Enfin, le processus d'apprentissage de l'utilisation de l'ACC semble mobiliser d'autres personnes que le conducteur et spécialement les professionnels des concessions automobiles (Aupetit, 2005 ; Jenness, et al., 2008 ; Portouli, \& Papakostopoulos, 2006).

Sur le plan méthodologique, Saad et al. (2004) pour le projet AIDE (2004-2008), entre autres, préconisent l'adoption d'une approche multi-niveaux de l'usage des SAC, permettant d'étudier de manière conjointe l'activité du conducteur considéré et ses interactions avec les autres usagers de la route. Cette ligne de recherche émerge, d'après AIDE, du manque de travaux empiriques intégrant d'autres individus que le conducteur « assisté ». Il s'agit de considérer davantage l'activité de conduite à un niveau individuel-collectif, dans la mesure où d'autres personnes contraignent par épisodes cette activité, ce qui fait que l'activité individuelle est en fait individuelle collective. Concrètement, au cours de l'usage du CCC s'articulent des périodes d'activité individuelle, où le conducteur est strictement mis en jeu, et des périodes d'activités individuelle-collectives, où interviennent les passagers et les autres usagers de la route, pour autant que ces derniers jouent un rôle significatif pour le conducteur. L'approche préconisée par Saad et al. (2004) et déjà appliquée dans Saad et al. (2006) et Lheureux et al. (2006) nécessite d'être développée de manière plus systématique. Au niveau méthodologique, le recueil de données exige ainsi de prendre en compte les collaborations entre l'ensemble des individus participant à l'action et la façon dont ils contraignent/facilitent l'activité du conducteur. Ce niveau d'analyse permet d'étudier conjointement les articulations individu-collectif sans « diluer » le conducteur dans le groupe et l'isoler de son environnement social. L'effort de recherche qui consiste à adopter, de manière systématique, ce type d'approche peut aboutir, à notre avis, à :

- Mieux comprendre comment d'autres personnes que le conducteur sont impliquées dans l'usage et l'appropriation du régulateur de vitesse conventionnel ;

- Caractériser l'activité des différents acteurs participant à la situation de conduite dans leurs rapports avec l'usage du régulateur ;

- Examiner l'usage du CCC en termes d'aide ou de gêne en considérant, au-delà du conducteur luimême, les systèmes sociaux « intra-véhiculaire » et « extra-véhiculaire ». 


\section{2.- Étudier les activités d'usage du régulateur conventionnel en situation « réelle »}

Une seconde piste d'enrichissement des recherches disponibles sur le CCC réside dans la prise en compte plus systématique de l'activité réelle des conducteurs (Ciaccabue, \& Saad, 2008; Dubart, Kassaagi, Popieul, Franc, \& Moessinger, 2008) : en (a) réduisant l'activité de conduite à une série de variables étudiées séparément, (b) l'étudiant dans des conditions expérimentales assez éloignées des situations de conduite réelles et (c) négligeant le point de vue subjectif des personnes engagées, ces recherches ont un potentiel de modélisation et d'explication limité.

\subsection{1.- Étude parcellaire v.s. étude intégrative de l'activité}

La plupart des recherches traitant du régulateur de vitesse conventionnel étudient les effets de l'utilisation du CCC sur une série de paramètres du comportement de conduite: vitesse, temps et distance intervéhiculaire, consommation de carburant, charge mentale... Le niveau d'analyse utilisé consiste à décomposer la tâche de conduite en éléments, fidèle en cela à la tradition ergonomique des «Human Factors », qui isole et étudie rigoureusement différents paramètres de la situation. Adopter ce niveau d'analyse a deux conséquences. La première est que cela engendre une compréhension parcellaire de l'usage du CCC, ne donnant pas accès à la globalité de l'activité du conducteur en interaction avec ce dispositif, l'activité humaine étant davantage que la juxtaposition des tâches qui la composent (Béguin, 2007). La seconde est que le fait d'examiner une activité dans son ensemble peut donner lieu à des résultats très différents de ceux qu'on obtiendrait en examinant isolément ses diverses composantes (Norman, 1993). Ainsi ces composantes, étudiées isolément, prennent une valeur globale d'explication du comportement de conduite: par exemple, la variation de la distance intervéhiculaire traduit à elle seule l'aspect sécuritaire de la conduite avec CCC. De sorte que la complexité de la conduite automobile est étudiée au moyen de la multiplication d'études rigoureuses des interactions entre différentes variables.

Une possibilité alternative est de prendre en compte l'activité de conduite automobile avec régulateur de vitesse dans sa globalité (Saad, \& Villame, 1996). La notion d'activité est ici considérée comme une totalité intégrant et combinant les émotions, les attentions, les perceptions, les actions, les communications, les focalisations et les interprétations des conducteurs (Villame, \& Theureau, 2001). Le passage par la notion d'activité offre la possibilité de ne pas dénaturer le comportement naturel du conducteur au profit d'une décomposition méthodologique, certes compréhensible. Le concept d'activité a pour objectif «de recoudre des parties de l'être humain auparavant séparées, disloquées pour [...] de « bonnes raisons philosophiques «» (Schwartz, 2007, p. 124). Il s'agit dans ce cas de mener des études intégratives de l'activité, c'est-à-dire qui décrivent la globalité de l'activité du conducteur. Au lieu de réduire l'usage du régulateur à la juxtaposition d'un certain nombre de variables, l'un des intérêts majeurs de cette approche est d'aborder les phénomènes dans leur complexité. Ce niveau d'analyse n'a pourtant été utilisé, d'après nous, que par Saad et al. (2006) pour étudier la conduite avec régulateur de vitesse conventionnel. L'adoption plus systématique d'une telle approche pourrait contribuer à:

- Caractériser les activités déployées par le conducteur en interaction avec son régulateur de vitesse conventionnel ;

- Identifier le poids pris par l'utilisation du régulateur de vitesse conventionnel dans l'ensemble des activités du conducteur;

- Montrer comment les aspects de l'activité motrice, de l'activité perceptive et de l'activité cognitive du conducteur et leurs relations sont significatifs pour la conduite.

\subsection{2.- Approche expérimentale v.s. étude en situation réelle}

Les travaux portant sur l'usage du CCC sur « route ouverte » restent encore peu nombreux (Lheureux, et al., 2006; Saad, et al., 2006; Young, \& Regan, 2007) face à celles concernant l'usage de 
l'ACC (Fancher, et al., 1998; Hoedemaeker, \& Kopf, 2001 ; Kopf, \& Nirschl, 1997; Kopf, \& Simon, 2001; McLaughlin, \& Serafin, 1999; Pio, \& McDonald, 2003; Saad, \& Villame, 1996; Ojeda, \& Nathan, 2006; Saad, \& Villame, 1999; Ward, Fairclough, \& Humphreys, 1995; Weinberger, Winner, \& Bubb, 2001). Néanmoins, l'essentiel des recherches pour ces deux systèmes est mené sur pistes privées (par exemple, Rudin-Brown, \& Parker, 2004; Smith, et al., 1994; Warren, 1982) ou sur simulateurs de conduite (notamment, Nilsson, 1995; Törnros, et al., 2002; Rajaonah, et al., 2006). Ces cadres expérimentaux contrôlés permettent (a) de stabiliser les conditions expérimentales et faire que l'ensemble des sujets soient confrontés à la même tâche, dans le même environnement, créant ainsi des conditions rigoureuses de comparaison, et (b) d'isoler les paramètres mesurés (variables dépendantes) du reste de la conduite (variables indépendantes).

Dans une approche «située » de la conduite automobile défendue dans Villame (2004), la situation de laboratoire est une situation culturelle parmi d'autres, s'inscrivant avec celles de terrain dans un continuum où les acteurs en présence sont « culturellement situés », et le chercheur ne jouit pas d'un statut «d'extraterritorialité » (Grison, \& Riff, 2002). Les situations sont susceptibles de se ranger dans des types distincts, constituant autant d'intermédiaires dans une continuité. Ce n'est donc plus la dichotomie naturel / artificiel qui est centrale, mais plutôt la notion de « situations d'étude privilégiées » (Grison, 1998; Testevuide, Riff, \& Grison, 2000); ces milieux contraints constituant des « isolats culturels » amplifient et mettent en évidence certaines facettes de l'activité des sujets. Il importe alors moins de promouvoir telle ou telle méthode scientifique, que de choisir la catégorie de situation la plus adéquate à l'étude de la catégorie de phénomène visée (Grison, \& Riff, 2002). Si les travaux de laboratoires portant sur le comportement de conduite avec CCC ont permis de générer des avancées considérables en matière de connaissances scientifiques, ces approches expérimentales présentent des limites dès lors que l'on souhaite envisager les intentions des conducteurs dans des contextes environnementaux dynamiques (Saad, et al., 2004) et fournir des préconisations pertinentes à la conception de système d'aide (Brézillon, 2006; Rajaonah, et al.., 2006). Dans ce cadre, l'étude de l'activité humaine, telle que décrite plus haut, nécessite forcément une analyse en situation dite « réelle » pour Haradji et Faveaux (2006), c'est-à-dire prise dans ses circonstances sociales, culturelles et techniques d'occurrence (Theureau, 1992): l'activité ne peut pas être abordée uniquement hors situation ou à partir d'une situation expérimentale qui réduirait l'activité, et/ou qui se développerait sans relation avec des études dans des situations non expérimentales (Theureau, 2001).

Un effort de recherche dans ce sens est actuellement perceptible au niveau européen concernant les études approfondies des nouveaux systèmes d'assistance à la conduite et dont les projets portant sur le régulateur de vitesse conventionnel pourraient s'inspirer. On peut citer le projet FESTA (2006-2008) dont l'objectif est de circonscrire une campagne de tests des répercutions de l'usage de technologies embarquées sur la conduite et qui met l'accent sur l'aspect « situé » de la conduite, en préconisant le recueil systématique d'un certain nombre d'éléments (type de routes empruntées, conditions de trafic, conditions météo...) venant compléter les autres données. Des préconisations qui seront mises en application dans le projet EuroFOT (2008-2010) et qui concernent, entre autres, l'ACC. Pour ce qui est de l'usage du CCC, disposer d'un corpus de données empiriques recueillies en contexte réel pourrait permettre de:

- Décrire l'activité déployée par le conducteur en relation avec son régulateur de vitesse conventionnel en conditions réelles de trafic ;

- Repérer les éléments de l'environnement matériel et social du conducteur qui contraignent l'usage du régulateur de vitesse en situation de conduite habituelle ;

- Identifier des typologies de situations de conduite quotidiennes qui posent des difficultés majeures au conducteur et qui sont liées à l'utilisation du CCC/

\subsection{3.- Données objectives sur le véhicule v.s. expérience subjective du conducteur}

Le corpus de données collectées dans les recherches portant sur l'usage du régulateur de vitesse conventionnel sont pour l'essentiel des données numériques relatives à la dynamique du véhicule 
(distance intervéhiculaire, temps intervéhiculaire, vitesse moyenne, distance de freinage...) recueillies à partir de capteurs embarqués. Un premier niveau de prise en compte de la subjectivité des conducteurs consiste à faire usage de questionnaires (Fancher, et al., 1995; Koziol, et al., 1999; Lheureux, et al., 2006; Rakha, et al., 2001; Young, \& Regan, 2007; Youngbin, 1997). Ce type de méthode est encore plus nettement répandu dans les travaux portant sur l'ACC (notamment, Hoedemaeker, \& Brookhuis, 1998; Hoedemaeker, \& Kopf, 2001; Nilsson, 1995; Nilsson, \& Nåbo, 1996; Rudin-Brown, \& Parker, 2004; Törnros, et al., 2002; Ward, et al., 1995; Ward, Humphreys, \& Fairclough, 1996 ). Ce premier niveau d'accès à la subjectivité est toutefois insuffisant pour rendre compte de façon acceptable d'éléments importants de l'expérience de conduite.

L'examen de la littérature disponible montre un manque d'investigations approfondies de l'expérience subjective de l'activité de conduite avec CCC (à l'exclusion de Saad et al., 2006 avec l'usage d'entretiens). Les futures recherches sur le modèle conventionnel pourraient ainsi s'inspirer de Saad et Villame (1996, 1999) ou Bjørkly, Jenssen, Moen, \& Vaa (2003), qui ont développé des outils méthodologiques destinés au recueil de données subjectives sur l'expérience de conduite régulée. Il serait également intéressant de tirer profit des avancées réalisées dans les projets FESTA et EuroFOT sur la prise en compte de données qualitatives relatives à l'expérience de conduite et, plus largement à l'expérience de conduite avec technologie embarquée telle qu'elle est analysée dans le cadre des «Field Operational Tests » (Fancher, et al., 1998; LeBlanc, Sayer, Winkler, Ervin, Bogard, Devonshire, et al., 2006; Weinberger, et al., 2001, par exemple).

L'ensemble de ces programmes de recherches récents semblent valider l'intérêt de la considération de la subjectivité des conducteurs, tant il est vrai que le point de vue de l'acteur apparaît comme un niveau d'analyse incontournable pour étudier l'activité (Bannon, 1991; Whiteside, \& Wixon, 1987). Ainsi, une description possible de l'activité de conduite nécessite la production de données objectives (du point de vue d'un observateur extérieur) sur le comportement de conduite et la dynamique du véhicule, recueillies à l'aide de capteurs par exemple, mais doit passer par le principe du primat de la description de l'expérience pour réussir à identifier les contraintes qui pèsent réellement sur le conducteur. Ce qui veut dire que ces données extérieures doivent être filtrées par le point de vue de l'acteur (Guérin, Riff, \& Testevuide, 2002) pour qu'elles puissent contribuer à documenter de manière rigoureuse l'activité de conduite. Considérer l'expérience subjective des conducteurs pourrait ainsi permettre d'enrichir la connaissance de l'usage du CCC sur plusieurs points: (a) accéder à des dimensions de l'activité inenvisageables avec les approches actuelles: les interprétations, les émotions, les sensations des conducteurs au cours de leurs expériences de conduite et le rapport entre elles, (b) identifier et documenter des difficultés y compris mineures, rencontrées par les usagers de SAC, qui, si elles ne conduisent pas à des conséquences graves contraignent néanmoins les acteurs dans leur conduite quotidienne, (c) identifier des éléments de l'environnement qui font signe pour le conducteur et qui déterminent ses modalités d'action. Grize (1995) montre comment la construction de cette signification résulte de l'interprétation qu'en fait l'individu. La signification est donc liée directement au conducteur et non observable avec un point de vue extérieur: "le conducteur est seul capable de nous fournir les éléments qui [...] vont nous permettre de comprendre [...] ce qu'il a pris en compte dans la situation, comment cela a contribué à la construction d'une action » (Villame, 2004, p. 158). Ces considérations impliquent un certain nombre de conséquences méthodologiques d'accès et de recueil de données concrètes quant à la prise en compte de cette subjectivité « située ». Le recueil approfondi des expériences subjectives de conduite avec régulateur de vitesse conventionnel pourrait permettre de:

- Décrire les dynamiques émotionnelles évoquées lors de l'usage du CCC ;

- Diagnostiquer les problèmes évoqués par le conducteur en relation avec ses expériences de conduite avec régulateur de vitesse conventionnel;

- Apprécier dans quelle mesure ces difficultés renvoient aux enjeux classiquement distingués en ergonomie : confort/inconfort, plaisir/déplaisir, appropriabilité/difficulté d'apprentissage, performance/baisse de performance, sécurité/risque ; 
- Discerner les éléments de l'activité de conduite liée à l'utilisation du régulateur de vitesse conventionnel qui sont mentionnés par le conducteur comme étant significatifs ;

- Repérer ce que le conducteur considère comme des erreurs, comment il les repère et les récupère.

\section{3.- Prendre en compte la caractéristique dynamique de l'usage du CCC}

L'examen de la littérature disponible a montré plus haut que la majorité des travaux concernant le régulateur de vitesse conventionnel n'accordait que peu d'importance à la dimension temporelle de l'usage du système, tant sur un plan théorique que méthodologique, excepté les deux études récentes menées dans AIDE (2004-2008). Les conclusions de Portouli et Papakostopoulos (2006) mettent d'ailleurs en avant l'absence de données empiriques sur les adaptations comportementales et les modifications de la conduite suscitées par un usage à long terme du CCC. Les recherches tendent à appréhender l'usage du régulateur comme si le temps n'était pas significatif, véhiculant l'idée générale d'une stabilité dans les comportements des utilisateurs. Tout se passe comme si les dispositifs méthodologiques utilisés « figeaient » le comportement du conducteur. Or les éléments apportés notamment par Saad et al. (2006) laissent clairement apparaître l'existence d'une « histoire d'usage » du système qu'il est nécessaire de prendre en compte dans la compréhension de la conduite avec CCC.

En premier lieu, l'usage et l'appropriation du régulateur de vitesse apparaissent comme des activités situées dynamiquement, c'est-à-dire faisant constamment appel aux ressources, individuelles comme collectivement partagées à divers degrés, que constituent les caractéristiques matérielles, sociales et culturelles changeantes de sa situation d'occurrence (Theureau, 2005, caractéristique énoncée pour toute activité humaine). Ces activités s'inscrivent dans des « histoires » qui sont fondamentalement dynamiques au sens où elles sont soumises à l'expérience acquise par les conducteurs (affinement des connaissances sur le fonctionnement du dispositif, plus grande maîtrise des commandes, identification des situations d'utilisation...) et aux changements perpétuels des situations de trafic rencontrées. Selon Proulx (2001), l'appropriation ne peut être comprise que dans le cadre d'un processus temporel impliquant des transformations de la situation par les utilisateurs. En second lieu, l'usage et l'appropriation du CCC ne sont pas des processus linéaires. L'action est considérée comme inscrite dans une dynamique faite d'états transitoires d'équilibres et de déséquilibres dont il s'agit de reconstruire l'histoire. Étudier le processus d'appropriation du régulateur revient à analyser cette histoire en repérant les éléments stabilisateurs ou déstabilisateurs, les ruptures et les connaissances, toujours en cours de constitution (Leblanc, Ria, Dieumegard, Serres, \& Durand, 2008, dans le cadre de l'enseignement professionnel).

Au niveau méthodologique, l'usage du CCC doit ainsi être analysé sur des périodes suffisamment longues pour permettre la description empirique de sa dynamique et de manière suffisamment systématique et fine pour reconstruire les perturbations dans cette activité. On ne peut se satisfaire de mesures ponctuelles de conduite pour comprendre ce processus complexe (ligne de recherche préconisée par FESTA, WP2, T2.3). Weinberger et al. (2001) et Ojeda et Nathan (2006), en développant des études sur des empans temporels plus long qu'à l'habitude, ont montré que deux à trois semaines d'utilisation intensive d'ACC (Weinberger, et al., 2001) et $400 \mathrm{~km}$ (Ojeda, \& Nathan, 2006) étaient nécessaires pour maîtriser le fonctionnement de ce dispositif et l'utiliser de manière adaptée dans le trafic. D'après Saad et al. (2004), la durée maximale d'une étude portant sur l'ACC est de quatre semaines consécutives (Fancher, et al., 1998; Weinberger, et al., 2001). Il apparaît évident que les futures recherches sur le régulateur de vitesse conventionnel gagnent à développer des approches qui prennent d'avantage en compte l'aspect dynamique de l'appropriation et l'usage de ce système en s'inspirant largement des études récentes concernant l'ACC. Considérer encore davantage cette composante dynamique de l'usage du CCC pourrait permettre, à notre avis, d'envisager les pistes suivantes :

- Modéliser la dynamique d'appropriation du régulateur de vitesse conventionnel en situation réelle; 
- Analyser en quoi le processus d'appropriation du système pose des difficultés au conducteur ;

- Apprécier dans quelle mesure le conducteur apprend sur le tas;

- Appréhender la manière dont se construisent et se déconstruisent les habitudes d'utilisation du CCC.

\section{Conclusion}

Cette analyse de la littérature a tenté de faire le point sur l'avancée des connaissances relatives à l'usage du régulateur de vitesse conventionnel. Au bilan, le résultat est contrasté. D'une part, les analyses montrent qu'on dispose d'un corps de connaissances intéressant sur les effets du CCC sur la conduite en terme de confort, de sécurité, de consommation, de coût cognitif, sur la modification des stratégies de conduites en terme d'activation/désactivation du régulateur, de gestion de la distance intervéhiculaire, de déclenchements des freinages, de gestion et de contrôle de vitesse. D'autre part, on a pu identifier plusieurs limites théoriques et méthodologiques à cette littérature qui ne permettent pas de donner accès à un certain nombre de situations d'usage du régulateur: la prise en compte insuffisante de la dimension collective de l'usage du régulateur, le manque de recherches menées sur l'activité complexe en situation réelle des conducteurs et la prise en compte insuffisamment développée de la dynamique d'usage des CCC.

Il nous semble qu'un effort important doit être mené sur le plan de la recherche dans l'étude de l'usage et l'appropriation du CCC, en tirant profit de la maturité des travaux portant sur le régulateur de distance, lorsque l'on sait qu'une tendance lourde de l'évolution des véhicules va dans le sens de l'implantation de ce type de système sur tous les segments du marché. La conduite avec régulateur va concerner dans un proche avenir la grande majorité des conducteurs y compris les plus vulnérables. Mais l'apport de cet article intéresse plus largement les programmes de recherches ergonomiques portant sur les SAC qui selon nous doivent intégrer plus radicalement des études en situation réelle de conduite. Les résultats de telles recherches sont susceptibles (1) d'orienter la conception des SAC et leur implantation dans le véhicule dans des perspectives qui allient confort et sécurité et donc en direction des constructeurs automobiles et des équipementiers de SAC, (2) de fournir des éléments de réflexion sur leur commercialisation par les constructeurs automobiles sur la base des expériences vécues et des difficultés des usagers, (3) d'identifier un certain nombre de pistes d'enrichissement de la formation continue des professionnels des auto-écoles concernant les difficultés d'apprentissage et d'usage des SAC que pourront expérimenter les apprentis conducteurs sur leur véhicule personnel après l'obtention du permis (en poursuivant les avancées réalisées dans le cadre du réseau d'excellence Humanist sur les relations entre ITS et formation à la conduite), (4) de générer un certain nombre d'avancées scientifiques portant sur la connaissance des activités du conducteur et ses interactions avec les dispositifs technologiques embarqués.

\section{REMERCIEMENTS}

Les auteurs tiennent à remercier Luciano Ojeda pour ses avis constructifs sur les versions successives de ce manuscrit et les évaluateurs de la revue Activités pour leurs commentaires critiques et suggestions.

\section{BIBLIOGRAPHIE}

AIDE (2004-2008). Adaptative Integrated Driver-Vehicle Interface. 6ème PCRD.

Aupetit, S. (2005). Conception d'aide à la dynamique d'appropriation des systèmes d'assistance à la conduite automobile : l'exemple du régulateur de vitesse. Mémoire de Master APCO (Activité Physique et Conception Ergonomique) non publié, Université d'Orléans. 
Bachmann, T., Naab K., Reichart, G., \& Scrault, M. (2000). Enhancing traffic safety with BMW's driver assistance approach connected drive ATA. Ingeneria Automotoristica, 53, 470-483.

Bannon, L. (1991). From human factors to human actors: the role of psychology and human-computer interaction studies in system design. In J. Greenbaum, \& M. Kyng (Eds.). Design at work: cooperative design of computer systems (pp. 25-44). Hillsdale: Erlbaum.

Barjonet, P. E. (1989). Sociologie(s) de la circulation et de la sécurité routière : quelques hypothèses pour un domaine de recherche. Recherche, Transports et Sécurité, 22, 43-48.

Béguin, P. (2007).Prendre en compte l'activité de travail pour concevoir. @ ctivités, 4(2), 107-114. http:// www.activites.org/v4n2/v4n2.pdf

Bjørkly, C.A., Jenssen, G.D., Moen, T., \& Vaa, T. (2003). Adaptive Cruise Control (ACC) and driver performance: effects on objective and subjective measures. Proceedings of the 10th World Congress on Intelligent Transport Systems, 16-20 nov 2003, Madrid, Spain.

Brézillon, J. (2006). Projet ACC : aide à la conduite basée sur le contexte. PREDIT GO3.

Cacciabue, P. C., \& Saad, F. (2008). Behavioural adaptations to driver support systems: a modelling and road safety perspective. Cognition, Technology \& Work, 10, 31-39.

Callahan, J. M. (1992). The cruise man. Automotive Industries, 172, 42-44.

Christ, R., Smuc, M., Gatscha, M., Schmotzer, C., \& Otzelberger, B. (2000). A field study on cruise control . On-line paper. Available: www.safexperiments.com/eng/main/ experimente.html

Connolly, T., \& Aberg, L. (1993). Some contagion models of speeding. Accident Analysis and Prevention, 25(1), 57-66.

De Waard, D. (1996). The measurement of drivers' mental workload. Doctoral dissertation. Retrieved March 30 2001, from Haren, The Netherlands: University of Groningen, Traffic Research Centre: http:/home. zonnet.nl/waard2/mwl.htm.

Dubart, D., Kassaagi, M., Popieul, J. C., Franc, M., \& Moessinger, M. (2008). Methodology to Improve ADAS. Specification Using Normal Driving Data. Proceedings of FISITA, World Automotive Congress, 14-19 September, Munich, Germany.

EuroFOT (2008-2009). European Field Operational Test on Active Safety Systems in vehicles. 7 ème PCRD.

Fancher, P., Baraket, Z., Johnson, G., \& Sayer, J. (1995). Evaluation of human factors and safety performance in the longitudinal control of headway. Proceedings of the World Congress on Intelligent Transport Systems, November 9-11, Yokohama, 1732-1738.

Fancher, P., \& Ervin, R. (1994). Implications of intelligent cruise control (ICC) systems for the driver's supervisory role. Proceedings of the ITS World Congress, Paris, France.

Fancher, P., Ervin, R., Sayer, J., Hagan, M.,. Bogard, S., Bareket, Z., Mefford, M., \& Haugen, J. (1998). The intelligent cruise control field operational test of the university of michigan for the US National Highway Traffic Safety Administration. Washington: Final Report.

FESTA (2006-2008). Field Operational Test Support Action. 7ème PCRD.

Givens, L. (1975). A primer on cruise controls. Automotive Engineering, 83(6), 26-32.

Goodman, M. J., Tijerina, L., Bents, F. D., \& Wierwille, W. W. (1999). Using cellular telephones in vehicles: safe or unsafe? Transportation Human Factors, 1, 3-42.

Grison, B. (1998). Structures de Raisonnement dans un laboratoire de Neurobiologie du Développement : étude dans une perspective d'Ecologie Cognitive. Paris: Thèse EHESS.

Grison, B., \& Riff, J. (2002). Validité écologique et situations d'étude privilégiées : de la psychologie expérimentale à l'anthropologie cognitive située. Actes des 4 èmes Journées d'Etudes de l'Association ACT'ING, Objets théoriques, objets de conception, objets d'analyse et situations d'étude privilégiées, 6-7 juin, Domaine de Chalès, Sologne. 
Grize, J. B. (1995). Signification. In M. de Montmollin (Ed.), Vocabulaire de l'ergonomie (pp. 99-112). Toulouse: Octarès Editions.

Guérin, J., Riff, J., \& Testevuide, S. (2002). Etude de l'activité «située» de collégiens en cours d'EPS : une opportunité pour réexaminer les conditions de validité des entretiens d'autoconfrontation. Revue Française de Pédagogie, 147, 15-26.

Haradji, Y., \& Faveaux, L. (2006). Évolution de notre pratique de conception (1985-2005) : modéliser pour mieux coopérer à partir des critères d'utilité, d'utilisabilité... @ ctivités, 3(1), 67-98. http://www.activites. org/v3n1/haradji.pdf

Hoedemaeker, M., \& Brookhuis, K. (1998). Behavioural adaptation to driving with an adaptive cruise control (ACC). Transportation Research Part F , 1, 95-106.

Hoedemaeker, M., \& Kopf, M. (2001). Visual sampling behaviour when driving with adaptive cruise control. Proceedings of the 9th International Conference on Vision in Vehicles, August 19-22, Australia.

Holland, C., \& Conner, M. (1996). Exceeding the speed limit, an evaluation of the effectiveness of a police intervention. Accident Analysis and Prevention, 28(5), 587-597.

Ioannou, P. A., \& Stefanovic, M. (2005). Evaluation of ACC vehicles in mixed traffic: lane change effects and sensitivity analysis. Intelligent Transportation Systems, 6(1), 79-89.

Jenness, J. W., Lerner, N. D., Mazor, S., Osberg, J. S., \& Tefft, B. C. (2008). Use of advanced in-vehicle technology by young and older early adopters. DOT HS 811 004, National Highway Traffic Safety Administration (NHTSA).

Kawano, A. (1992). Cruise control device for motor vehicles. Patent 5129475.

Keegan, D. (1996). Road citizenship. Recovery, 7(2), 1380-1383.

Kopf, M., \& Nirschl, G. (1997). Driver-vehicle interaction while driving with ACC in borderline situations. Proceedings of the Fourth World Congress on Intelligent Transport Systems, Berlin, Germany.

Kopf, M., \& Simon, J. (2001). A concept for a learn-adaptive advanced driver assistance system. Proceedings of the Conference on Cognitive Science Approaches, Neubiberg, September.

Koziol, J. S., Inman, V. W., Carter, M., Robinson, M., \& Barker, M. (1999). Evaluation of intelligent cruise control system. Volume 1 - Study results (DOT-VNTSC-NHSTA-98-3). Washington, DC: Department of Transport.

LeBlanc, D., Sayer, J., Winkler, C., Ervin, R., Bogard, S., Devonshire, J. Mefford, M., Hagan, M., Bareket, Z., Goodsell, R., \& Gordon, T. (2006). Road departure crash warning system field operational test. University of Michigan for the US National Highway Traffic Safety Administration, Washington. Final Report.

Leblanc, S., Ria, L., Dieumegard, G. Serres G., \& Durand M. (2008). Concevoir des dispositifs de formation professionnelle des enseignants à partir de l'analyse de l'activité dans une approche enactive. @ ctivités, 5(1) 58-78. http://www.activites.org/v5n1/v5n1.pdf

Lee, J. D., Caven, B., Haake, S., \& Brown, T. L. (2001). Speech-based interaction with in-vehicle computers: the effect of speech-based e-mail on drivers' attention to the road. Human Factors, 43(3), 631-640.

Lheureux, F., Saad, F., Pianelli, C., Abric, J. C., \& Roland, J. (2006). Behavioural changes due to long term use of speed limiter and cruise control. In AIDE project (Adaptive Integrated Driver-vehicle Interface), D1.2.4., Long-term phase test and results (pp. 39-64). European Commission, Brussels.

Ma, R., \& Kaber, D. B. (2005). Situation awareness and workload in driving while using adaptive cruise control and a cell phone. International Journal of Industrial Ergonomics, 35(10), 939-953.

Malaterre, G., \& Saad, F. (1986). Les aides à la conduite : définitions et évaluation. Exemple du radar anticollision. Le Travail Humain, 49(4), 333-346.

McLaughlin, S, \& Serafin, C. (1999). Measurement of driver intervention responses during transition from ACC deceleration to manual control. Proceedings of the ITS America Conference, Washington DC, USA. 
Nilsson, L. (1995). Safety effects of adaptive cruise controls in critical traffic situations. Proceedings of the second World Congress on Intelligent Transport Systems, Yokohama, Japan, November 9-11, 1254-1259.

Nilsson, L., \& Nåbo, A. (1996). Evaluation of application 3: intelligent cruise control simulator expriment. VTI särtryck No 266, Linköping, Sweden.

Norman, D. A. (1993). Les objets dans l'action. Raisons Pratiques, 4, 15-34.

Ojeda, L., \& Nathan, F. (2006). Studying learning phases of an ACC through verbal reports. In R.F.T. Brouwer, \& D.M. Hoedemaeker (Eds.), Driver support and information systems: experiments on learning, appropriation and effects of adaptiveness (pp. 47-73). AIDE project (Adaptive Integrated Driver-vehicle Interface), D1.2.3. European Commission, Brussels.

Patterson, A. K. (1998). Intelligent cruise control system impact analysis. Unpublished Masters thesis, Virginia Polytechnic Institute and State University.

Peretti-Watel, P. (2001). La conduite automobile : un objet de recherche sociologique ? European Journal of Sociology, 42, 391-428.

Pio, J., \& McDonald, M. (2003). Stop-and-Go behaviour: initial findings from floating vehicle trials. Proceedings of the $10^{\text {th }}$ world congress on Intelligent Transport Systems and Services, Madrid, Spain .

Portouli, V., \& Papakostopoulos, V. (2006). On-road study on short-term effects of ADAS on driving behaviour. In R.F.T. Brouwer, \& D.M. Hoedemaeker (Eds.), Driver support and information systems: experiments on learning, appropriation and effects of adaptiveness (pp. 74-96). AIDE project (Adaptive Integrated Driver-vehicle Interface), D1.2.3. European Commission, Brussels.

Prestl, W., Sauer, T., Steinle, J., \& Tschernoster, O. (2000). The BMW active cruise control ACC. SAE Technical paper.

Proulx, S. (2001). Usages de l'Internet : la « pensée-réseaux » et l'appropriation d'une culture numérique. In E. Guichard (Ed.), Comprendre les usages de l'Internet (pp. 139-145). Paris: Éditions Rue d’Ulm, ENS.

Rajaonah, B., Anceaux, F., \& Vienne, F. (2006). Trust and the use of adaptive cruise control: a study of a cutin situation. Cognition, Technology and Work, 8(2), 146-155.

Rakha, H., Hankey, J., Patterson, A., \& Van Aerde, M. (2001). Field evaluation of safety impacts of adaptive cruise control. ITS Journal, 6, 1-35.

Regan, M. A., \& Mitsopoulos, E. (2003). Understanding passenger influences on driver behaviour: implications for road safety and recommendations for countermeasure development. Monash (Australia): Monash University Accident Research Centre.

Regan, M. A., \& Young, K. L. (2004). Use of manual speed alerting and cruise control dives by driver in New South Wales. Monash (Australia): Monash University Accident Research Centre.

Renault (2004). Guide de mise en main du régulateur/limiteur de vitesse.

Rudin-Brown, C. M., \& Parker, H. A. (2004). Behavioural adaptation to adaptive cruise control (ACC): implications for preventive strategies. Transportation Research, 7, 59-76.

Saad, F., Dionisio, C., Arslanian, E., Collaciuri, V., Legay, F., Vézier, B., \& Timianguel, J. (2006). Learning phase and short term behavioural effect of speed limiter and cruise control systems. In R.F.T. Brouwer, \& D.M. Hoedemaeker (Eds.), Driver support and information systems: experiments on learning, appropriation and effects of adaptiveness (pp. 19-46). AIDE project (Adaptive Integrated Driver-vehicle Interface), D1.2.3. European Commission, Brussels.

Saad, F., Hjälmdahl, M., Cañas, J., Alonso, M., Garayo, P., Macchi, L., Nathan, F., Ojeda, L., Papakostopoulos, V., Panou, M., \& Bekiaris, E. (2004). Behavioural effects of driver assistance system. AIDE project (Adaptive Integrated Driver-vehicle Interface), D1.2.1. European Commission, Brussels.

Saad, F., \& Malaterre, G. (1982). Régulation de la vitesse, aide au contröle de la vitesse. Rapport ONSER.

Saad, F., \& Villame, T. (1996). Assessing new driving support system: contribution of an analysis of drivers' activity in real situations. Third annual World Congress on Intelligent Transport Systems, Orlando, USA. 
Saad, F. \& Villame, T. (1999). Intégration d'un nouveau système d'assistance dans l'activité des conducteurs d'automobile. In J.G. Ganascia (Ed.), Sécurité et cognition, (pp. 105-114). Paris: Editions Hermès.

Sayer, J. R., Mefford, M. L., Shirkley, K., \& Lantz, J. (2005). Driver distraction: a naturalistic observation of secondary behaviors with the use of driver assistance systems. Proceedings of the Third International Driving Symposium on Human Factors in Driver Assessment, Training and Vehicle Design, June 27-30, Rockport, Maine, USA, 262-268.

Scapaticci, D., Fermi, U., \& Saroldi, A. (2000). Safety and comfort driver support functions in Tripmate concept car ATA. Ingeneria Automotoristica, 53, 466-469.

Schober, M., Eskandarian, A., Kühne, R. D., \& Wehlan, H. (2004). Comparing driving behaviour for manual, conventional and automated cruise control driving in car-following by scenarios based on an advanced driver performance map. Proceedings of the ITS Safety and Security Conference, Miami, US.

Schwartz, Y. (2007). Un bref aperçu de l'histoire culturelle du concept d'activité. @ ctivités, 4(2), 122-133. http://www.activites.org/v4n2/v4n2.pdf

Shaout, A., \& Jarrah, M. A. (1997). Cruise control technology review. Computers and Electrical Engineering, $23,259-271$.

Smith, F. J., Ashby, M. C., \& Fairclough, S. H. (1994). Cruise control use: driver attitudes and behaviour. Proceedings of the Triennial Congress of the International Ergonomics Association, Toronto, 252-254.

Stanton, N. A., \& Young, M. S. (2005). Driver behaviour with adaptive cruise control. Ergonomics, 48(10), 1294-1313.

Stanton, N. A., Young, M. S., \& McCaulder, B. (1997). Drive-by-wire: the case of driver workload and reclaiming control with adaptive cruise control. Safety Science, 27, 149-159.

Szuszman, I. P. (2005). Adaptive cruise control : system overview. Proceedings of the $5^{\text {th }}$ Meeting of the U.S. Software System Safety Working Group, 12-14 avril, Anaheim, CA, USA.

Testevuide, S., Riff, J., \& Grison, B. (2000). La situation d'étude privilégiée : une notion opératoire pour un dialogue fructueux entre recherche et intervention. Actes du Colloque ARIS, Grenoble, 14-16 décembre, $39-42$.

Theureau, J. (1992). Le Cours d'Action : analyse sémio-logique. Essai d'une anthropologie cognitive située. Berne: Peter Lang.

Theureau, J. (2001). Note sur l'histoire des «émotions» dans l'étude du cours d'action. France-Télécom, 15 Novembre, Paris.

Theureau, J. (2005). Les méthodes de construction de données du programme de recherche sur les cours d'action et leur articulation collective, et ... la didactique des activités physiques \& sportives ?, Impulsion, 4, 281-301.

Theureau, J., \& Jeffroy, F. (2004). Ergonomie des situations informatisées : la conception centrée sur le cours d'action des utilisateurs. Toulouse: Octarès Editions.

Törnros, J., Nilsson, L., Östlund, J., \& Kircher, A. (2002). Effects of ACC on driver behaviour, workload and acceptance in relation to minimum time headway. Proceedings of the 9th World Congress on Intelligent Transport Systems, 14-17 October, Chicago, USA.

Travis, C. J. (1982). Cruise control improves fuel mileage. Diesel Equipment Superintendent, 60(4), 40.

Turrentine, T., Sperling, D., \& Hungerford, D. (1991). Consumer acceptance of adaptive cruise control and collision avoidance systems. Transportation Research Record, 1318, 118-121.

Van Kampen, L. T. B. (1996). Cruise control in personenauto's: een literatuur-orientatie op verkeersveiligheidsaspecten. Report $\mathrm{n}^{\circ} \mathrm{R}-96-21$, SWOW, The Netherlands.

Villame, T. (2004). Conception de systèmes d'assistance au conducteur : comment prendre en compte le caractère complexe, dynamique et situé de la conduite automobile ? Cognition située et conception de systèmes d'assistance au conducteur. @ctivités, 1(2), 146-169. http://www.activites.org/v1n2/villame.pdf 
Villame, T., \& Theureau, J. (2001). Contribution of a 'comprehensive analysis' of human cognitive activity to the advanced driving assistance devices design. In R. Onken (Ed.), Proceedings of CSAPC'01, $8^{\text {th }}$ Conference on Cognitive Science Approaches to Process Control (pp. 23-26). Bonn: DGLR.

Wang, J. S., Knipling, R. R., \& Goodman, M. J. (1996). The role of driver inattention in crashes: new statistics from the 1995 Crashworthiness Data System. Proceedings of the 40th Annual Association for the Advancement of Automotive Medicine, Vancouver, British Columbia, 377-392.

Ward, N. J., Fairclough, S., \& Humphreys, M. (1995). The effect of task automatisation in the automotive context: a field study of an autonomous intelligent cruise control system. In D.J. Garland, \& M.R. Endsley (Eds.), Experimental Analysis and Measurements of Situational Awareness (pp. 369-374). Daytona Beach, Florida, USA: Embry-Riddle Aeronautical University Press.

Ward, N. J., Humphreys, M., \& Fairclough, S. (1996). A field study of behavioural adaptation with an autonomous intelligent cruise control system. Proceedings of the International Conference on Traffic and Transport Psychology, 22-25 May, Valencia, Spain, 15-19.

Warren, D. L. (1982). Speed zoning and control. Synthesis of safety research related to traffic control and roadway elements. Chapter 17. FHA, Washington, D. C.

Watanabe, T., Kishimoto, N., Hayafune, K., \& Yamada, K. (1995). Development of an ICC system. Proceedings of the World Congress on Intelligent Transport Systems, Yokohama, 1229-1235.

Weinberger, M., Winner, H., \& Bubb, H. (2001).Adaptive cruise control field operational test: the learning phase. JSAE Review, 22, 487-494.

Whiteside, J., \& Wixon, D. (1987). Improving human-computer interaction - a quest for cognitive science. In J. M. Carroll (Ed.). Interfacing thought - Cognitive aspects of human-computer interaction (pp. 353-365). Cambridge: MIT Press.

Young, K. L., \& Regan, M. A. (2007). Use of manual speed alerting and cruise control devices by car drivers. Safety Science, 45(4), 473-485.

Young, M. S., \& Stanton, N. A. (1997). Automotive automation: investigating the impact on drivers' mental workload. International Journal of Cognitive Ergonomics, 1, 325-336.

Youngbin, Y. (1997). A focus group study of automated highway systems and related technologies. Berkley (California): California PATH Working Paper, Institute of Transport Studies.

Zaidel, D. M., Paarlberg, W. T., \& Shinar, D. (1978). Driver performance and individual differences in attention and information processing. Volume 1: Driver inattention. Institute for Research in Public Safety, DOT-HS-803 793.

RÉSUMÉ

Cet article propose une revue critique de la littérature scientifique disponible sur l'usage du régulateur de vitesse « conventionnel » (CCC pour Conventional Cruise Control). Ce système d'assistance à la conduite, qui prend en charge le maintien de la vitesse du véhicule, intègre progressivement l'ensemble des gammes de modèles automobiles. L'analyse de la littérature permet (1) de mettre en évidence les effets positifs identifiés du CCC sur l'activité de conduite et les problèmes liés à son utilisation, (2) de souligner le manque d'études systématiques de type ergonomique sur son usage, malgré les efforts des équipementiers et des constructeurs automobiles pour diffuser ce dispositif sur l'ensemble des segments du marché, (3) d'identifier des perspectives d'enrichissement de ces études aux niveaux de la dimension individuelle-collective de l'usage du régulateur, des conditions de recueil des données laboratoire-situations réelles, et du caractère statique-dynamique de l'appropriation du CCC. Ces perspectives tirent profit de la maturité des recherches sur le « régulateur de distance »(ACC pour Adaptive Cruise Control), version évoluée du modèle conventionnel qui a, lui, donné lieu à une littérature scientifique plus riche malgré son apparition récente. 


\section{MoTS CLÉS}

Système d'assistance à la conduite automobile ; Ergonomie ; Régulateur de vitesse conventionnel ; Action située

\section{RÉFÉRENCES}

Aupetit, S. \& Riff, J. (2010). Approche ergonomique de l'usage du régulateur de vitesse conventionnel : analyse critique de la littérature scientifique, Activités, 7(2), 105-123. http://www.activites.org/v7n2/v7n2. pdf.

Article soumis le 26 Janvier 2009, accepté pour publication le 30 juin 2010. 